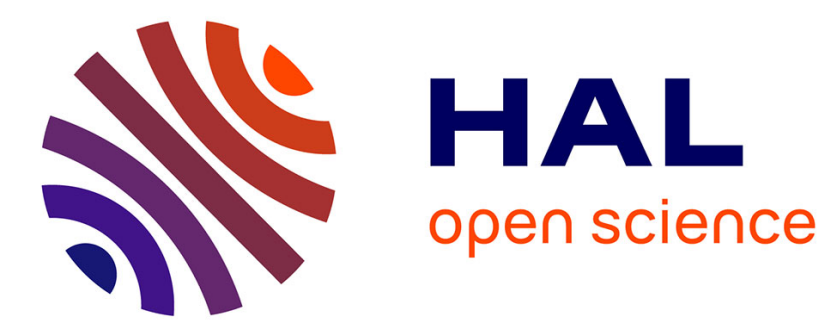

\title{
Sceaux des musées de Kayseri et de Niğde (Cappadoce byzantine)
}

\author{
Sophie Métivier
}

\section{To cite this version:}

Sophie Métivier. Sceaux des musées de Kayseri et de Niğde (Cappadoce byzantine) . Studies in Byzantine Sigillography, 2010, 10, p. 61-74. halshs-01351220

\section{HAL Id: halshs-01351220 \\ https://shs.hal.science/halshs-01351220}

Submitted on 4 Nov 2016

HAL is a multi-disciplinary open access archive for the deposit and dissemination of scientific research documents, whether they are published or not. The documents may come from teaching and research institutions in France or abroad, or from public or private research centers.
L'archive ouverte pluridisciplinaire HAL, est destinée au dépôt et à la diffusion de documents scientifiques de niveau recherche, publiés ou non, émanant des établissements d'enseignement et de recherche français ou étrangers, des laboratoires publics ou privés. 


\section{Sophie Métivier}

\section{SCEAUX DES MUSEES DE KAYSERI ET DE NIĞDE (CAPPADOCE BYZANTINE)}

Je présente ici les dix-neuf sceaux (ou tessère) que j'ai examinés et photographiés dans les musées de Kayseri et de Niğde en septembre 2004. Je prie Monsieur Hamdi Biçer, directeur du musée de Kayseri, et Monsieur Fazıl Açıkgöz, directeur du musée de Niğde, de trouver ici l'expression de mes remerciements. La moderne Kayseri recouvre l'ancienne métropole de Cappadoce Première, Césarée, tandis que Niğde est peut-être attestée dès la fin du VIII ${ }^{e}$ siècle dans les sources arabes sous le nom de Māğida à $15 \mathrm{~km}$ au nord de la métropole de la Cappadoce Seconde, Tyane. ${ }^{1}$

\section{Sceaux du musée de Kayseri}

Le musée de Kayseri abrite trois sceaux et une tessère. N'ayant pas eu accès aux registres d'inventaire des fouilles, je ne suis pas sûre d'avoir vu la totalité des sceaux du musée de Kayseri. Ceux que j'ai repérés étaient inventoriés par erreur avec les monnaies. Ces quatre pièces datent du $\mathrm{XI}^{\mathrm{e}}$ siècle, l'une au moins se rapporte à la Césarée byzantine.

\section{Aarôn, protospathaire, épi tou chrysotriklinou...}

Inv. : $71 / 102-11$

Dia. : 2,6 - 2,8. Sceau légèrement rogné. Frappe un peu décentrée. Lettres écrasées. Inédit.

Au droit, dans un cercle de perles, buste de saint militaire avec une barbe longue, en costume militaire, avec lance et bouclier bordé d'un cercle de grosses perles. Dans le champ, inscription en colonne à gauche et à droite de l'effigie:

$$
\begin{aligned}
& \text { ब( }|\Theta \in| 0|\Delta| \omega \ldots \\
& \text { O } \propto \text { ( }(\gamma 10 \varsigma) \Theta \varepsilon o ́ \delta \omega[\rho \circ \varsigma]
\end{aligned}
$$

Au revers, inscription sur six lignes précédée d'une croix cantonnée de perles et accostée de tirets; cercle de perles.

1 Fr. Hild et M. Restle, Kappadokien (Kappadokia, Charsianon, Sebasteia und Lykandos) (Österreichische Akademie der Wissenschaften. PhilosophischHistoriche Klasse, Denkschriften, 149. TIB, 2) (Vienne, 1981), s.v. Nakīdā, 243244. Māğida, localisée par al-Mas ' ūdī à $20 \mathrm{~km}$ de Loulon dans la région des mațāmir, est mentionnée pour la première fois par al-Ṭabarī à l'occasion de sa prise par Hārūn al-Rashīd en 782. 


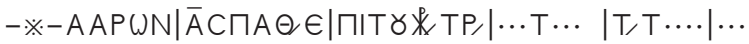

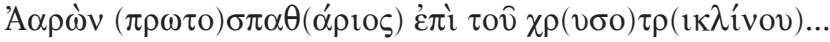
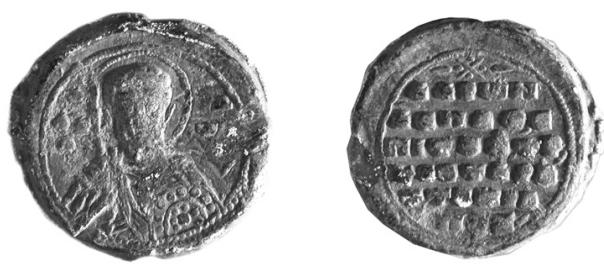

Plusieurs sceaux de Aarôn avec buste de saint Théodore, contemporains de celui-ci, sont connus. Ils sont attribués au prince bulgare, beau-frère d'Isaac, le premier empereur de la dynastie des Comnènes. Contrairement au présent sceau, ils présentent tous le formulaire Kúpıє ßoฑ́ $\theta \varepsilon \imath$ : SBS 8 (2003), 161, 195 ; SBS 6 (1999), 103 ; SBS 5 (1998), 109, 144, 197. V. Šandrovskaja a édité plusieurs sceaux ayant appartenu à cet officier. ${ }^{2}$

Datation : second quart du $\mathrm{XI}^{\mathrm{e}}$ siècle.

\section{Basile Pédiaditès}

Inv. : 76/78.

Dia. : 22,3 - 23,5. Sceau échancré aux deux extrémités du canal, légèrement rogné et oxydé.

Inédit.

Au droit, saint en buste, de face, aux cheveux courts et avec chlamyde. Dans le champ, inscription en colonne, dont quelques lettres sont lisibles à droite :

$$
\begin{aligned}
& \text { K.이미 }
\end{aligned}
$$

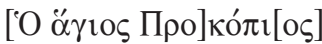

Il s'agit probablement de saint Procope étant donné le type iconographique du saint. ${ }^{3}$

Au revers, inscription sur cinq lignes, précédée, à la première ligne,

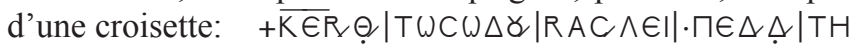

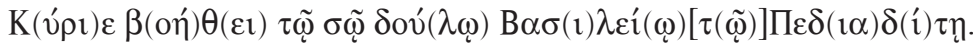
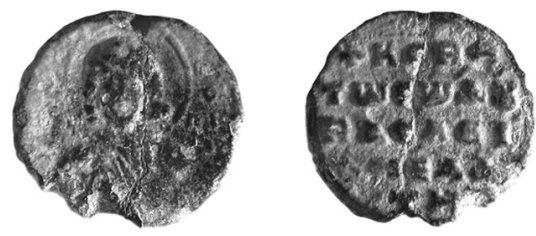

2 V. Šandrovskaja, « Neizvestnye pečati Aarona, magisra i duki Iverii i Velikoj Armenii i proedra i duki XI v (sceaux inconnus de Aaron, magistre et duc d'Ibérie et de Grande Arménie et du même, proèdre et duc), » Soobščenija Gosudarstvennogo Ermitaža 37 (1973) 60-64.

3 Voir, par exemple, Laurent, Corpus V/1, n 211. 
Datation : première moitié du $\mathrm{XI}^{\mathrm{e}}$ siècle. Cette datation écarte toute identification avec le lettré Basile Pédiaditès, diacre, maître des rhéteurs et métropolite de Corfou, aux $\mathrm{XII}^{\mathrm{e}}$ - XIII ${ }^{\mathrm{e}}$ siècles. ${ }^{4}$ En revanche un homonyme et contemporain est connu comme catépan de Sicile en 1040 - 1041 grâce à Kékauménos et Skylitzès ainsi que comme catépan d'Antioche, probablement entre 1037 et 1040, grâce à un sceau de la collection Zacos de la Bibliothèque nationale de France édité par Jean-Claude Cheynet. ${ }^{5}$ Faut-il identifier le Basile Pédiaditès du sceau de Kayseri avec le catépan de Sicile et d'Antioche ? Au droit du sceau de ce dernier, on a un buste de saint Michel, ayant en main un globe et un sceptre, vêtu d'un loros, désigné par son nom dans le champ. L'effigie est accompagnée de l'inscription circulaire : $K(v ́ \rho \imath) \varepsilon \beta o \eta ́ \theta \varepsilon \imath \tau \hat{~} \sigma \hat{\varphi}$ $\delta(o v ́ \lambda \omega)$. Les lettres encore visibles de la légende et l'iconographie du sceau de Kayseri excluent qu'il s'agisse de saint Michel.

\section{Eugénios, métropolite de Césarée (?)}

Inv. : $77 / 573$.

Dia. : 23,3/25. Sceau échancré aux extrémités du canal et rogné sur la gauche, entaillé au revers. Lettres écrasées.

Inédit.

Au droit, saint évêque en buste de face, bénissant de sa main droite, Livre dans la main gauche. Inscription en colonne de part et d'autre de l'effigie : à gauche, plusieurs lettres; à droite, $\wedge|\in \|| \mathrm{OC}$.

[OO ö $\gamma 10 \varsigma \mathrm{B} \alpha \sigma i] \lambda \lambda \varepsilon 10$.

Au revers, inscription métrique sur huit lignes, précédée d'une croisette au début de la première ligne.
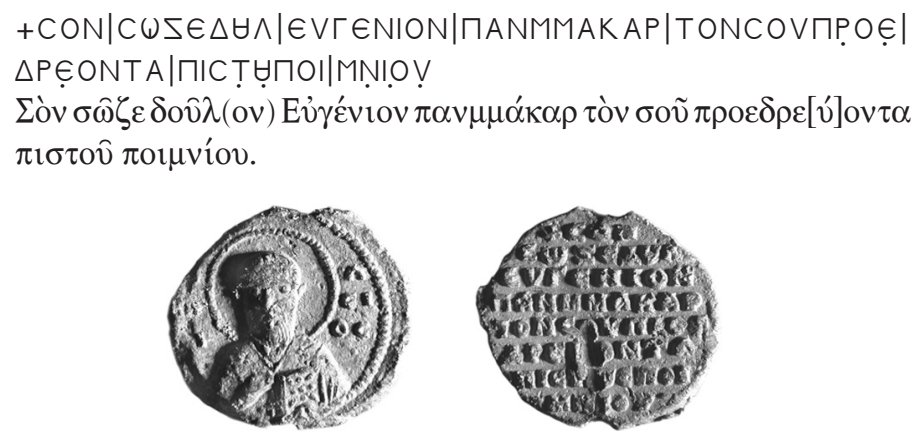

Datation : seconde moitié du $\mathrm{XI}^{\mathrm{e}}$ siècle.

4 R. Browning, « The Patriarchal School at Constantinople, » Byz. 33 (1963) 20-22.

5 Voir J. - C. Cheynet, «Les ducs d'Antioche sous Michel IV et Constantin IX, » Novum Millennium. Studies on Byzantine History and Culture dedicated to Paul Speck, éd. C. Sode et S. Takacs (Aldershot, 2001), 54-55. Vente aux enchères Spink 135, Londres, 6 octobre 1999, n²60, cité dans BZ 93 (2000) 397, par W. Seibt : sceau d'un Basile Pédiaditès, catépan d'Antioche. 
L'invocation mentionne Eugénios, proèdre du troupeau du saint. S'agitil du métropolite de Césarée, successeur de saint Basile, ou de l'higoumène d'un monastère Saint-Basile ${ }^{6}$ ? On connaît un exemple sigillographique qui associe l'image de saint Basile à un évêque de Césarée qui ne soit pas homonyme. La collection de Dumbarton Oaks conserve le sceau de Stylianos, archevêque de Césarée et syncelle, attesté en 1049, qui comporte au droit l'effigie de saint Basile en buste. ${ }^{7}$ Le décret synodal sur la nomination aux évêchés du 9 novembre 1071 mentionne parmi les évêques présents le prôtothronos Eugénios de Césarée, qui n’est pas autrement connu. ${ }^{8}$ Ce sceau de Kayseri lui a vraisemblablement appartenu. Pour le $\mathrm{XI}^{\mathrm{e}}$ siècle on ne connaît nommément que trois titulaires de l'évêché de Césarée, Léon en 997, Stylianos en 1049 et ce dernier Eugénios. ${ }^{9}$

\section{Tessère de Tornikios proèdre}

Inv. : 77/491.

Dia. : manquant. Sceau légèrement échancré et rogné. Lettres écrasées.

Éd. des parallèles : W. Seibt, Sammlung Köhler-Osbahr, t. V, 4, Byzantinische Münzen und ihr Umfeld. Bleisiegel und -plomben sowie Gewichte aus dem östlichen Mittelmeerraum (Duisburg, 2001), n 47 (copie). SBS 8 (2003), 219 (Gorny, Vente 89, 5 mai 1998, 570). Jean-Claude Cheynet me signale trois sceaux similaires inédits : Zacos (BnF) 1032, 1033, 1034.

Au droit, deux saints en pied, de profil, se tenant embrassés. Inscriptions

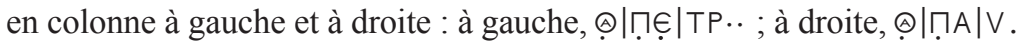

'O $\ddot{\alpha}(\gamma 10 \varsigma) \Pi \varepsilon \dot{\tau} \rho[\mathrm{o}$ ]

'O $\ddot{\alpha}(\gamma 10 \varsigma) \Pi \alpha \tilde{v}[\lambda \circ \varsigma]$

Au revers, inscription sur sept lignes, légende métrique de 12 syllabes :

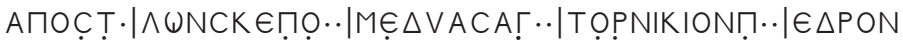

$\in K \sqcap \cdot|\underline{H} H C \underline{B} \wedge A \cdot| H C$

6 La forme verbale $\pi \rho{ } \delta \rho \varepsilon v ́ \omega$ est employée pour désigner Euthyme comme évêque de Sardes dans Skylitzès, Michel II, section 4, 1. 16, pour mentionner le patriarche de Constantinople dans Jean Zonaras, Epitomae historiarum, éd. M. Pinder (Bonn 1897) (CSHB), t. 3, 261, 1. 7. Si je ne connais pas de parallèle à cet emploi sur les sceaux, le substantif $\pi \rho o ́ \varepsilon \delta \rho o \varsigma$ est bien attesté : voir Laurent, Corpus, V/1, p. xxxi.

7 DOSeals 4.41.2. Je ne connais pas d'autres exemples de sceaux d'évêques de Césarée de Cappadoce avec l'effigie de saint Basile. Les autres sceaux d'évêques de Césarée, mentionnés dans V. Laurent, Corpus V/1 et V/3 et dans DOSeals vol. 4 , sont antérieurs au milieu du $\mathrm{x}^{\mathrm{e}}$ siècle.

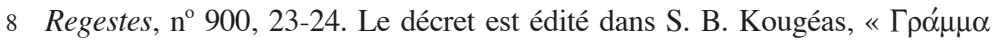

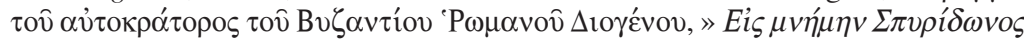
$\Lambda \alpha ́ \mu \pi \rho o v$, (Athènes, 1935), 574-575.

9 Voir G. Fedalto, Hierarchia Ecclesiastica Orientalis (Padoue, 1988), I, 22, qui omet Stylianos. 


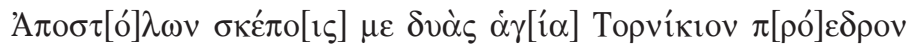

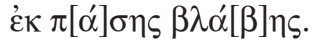
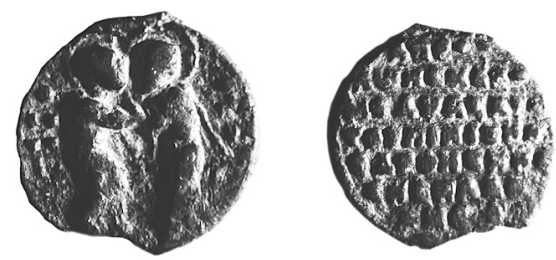

Datation proposée : seconde moitié du $\mathrm{XI}^{\mathrm{e}}$ siècle.

\section{Sceaux du musée de Niğde}

Les quinze sceaux conservés au musée de Niğde, dont on ignore les conditions de découverte, sont antérieurs au $\mathrm{IX}^{\mathrm{e}}$ siècle, à une exception près. Il s'agit de deux sceaux à monogrammes compacts, de deux à monogrammes cruciformes, de trois à inscriptions bilatérales et pour le reste de sceaux à monogrammes invocatifs. Quatre dignités différentes sont mentionnées : deux apo éparchôn, deux apo hypatôn, deux stratélates, un patrice. Deux fonctions sont indiquées, celles d'évêque et de commerciaire. Dignités et fonctions sont donnés séparément l'une de l'autre.

\section{Jean, apo hypatôn}

Inv. : 1.46 .98 .

Dia. : 24,5. Sceau échancré aux deux extrémités au droit, légèrement rogné. Lettres bien frappées.

Inédit.

Au droit, inscription sur quatre lignes, précédée d'une croisette ; cercle de feuilles. Ligature des deux $N$.

$+|\omega| A N N O Y|A \sqcap O Y \Pi A| T \omega N$

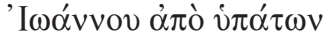

Au revers, inscription sur quatre lignes, peut-être précédée d'une croisette :

$+\triangle O Y|\wedge O Y T H C| \Theta \in O T O \mid K O Y$

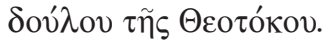
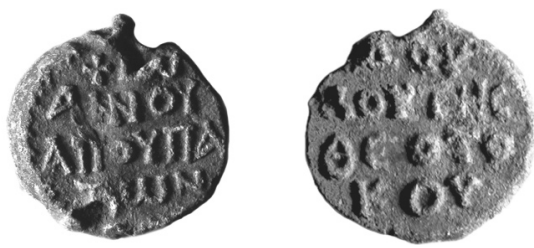

Datation : $\mathrm{VI}^{\mathrm{e}} / \mathrm{VII}^{\mathrm{e}}$ siècle. 


\section{Marinos}

Inv. : 1.50 .98 .

Dia. : 17,7. Sceau échancré aux deux extrémités. Lettres bien gravées. Inédit.

Au droit, monogramme compact avec A, $M$ et $N$ dans le carré, $\succ$ au sommet à gauche, $P$ en haut à droite; une étoile à gauche, sans doute une

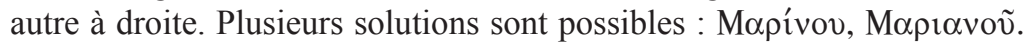
Compte tenu du revers, la première, Mapívov, doit être retenue.

Au revers, inscription sur deux lignes ; cercle de feuilles.

MAP|INC

Mapívov.
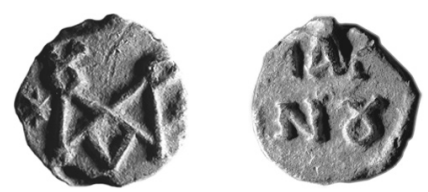

Datation : $\mathrm{VI}^{\mathrm{e}}$ siècle/VII ${ }^{\mathrm{e}}$ siècle.

\section{Sisinnios, apo éparchôn}

Inv. : 1.48 .98 .

Dia. : 18,6. Sceau échancré aux deux extrémités du canal et légèrement rogné. Lettres bien frappées.

Parallèles : Trois exemplaires à Berlin.

Éd. : Sode, Bleisiegel, $\mathrm{n}^{\text {os }}$ 189, 190 et 191 ; SBS 3 (1993), 200.

$\mathrm{Au}$ droit, monogramme compact avec $\mathrm{C}$ et $\mathrm{N}$ dans le carré, $\succ$ en haut à droite ; cercle de feuilles.

Sioıvvíov

Au revers, monogramme compact avec A, N, $\Pi$ et $X$ dans le carré, $\omega$ accroché à la partie inférieure de la haste gauche, $P$ et $E$ à droite ; cercle de feuilles.

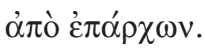
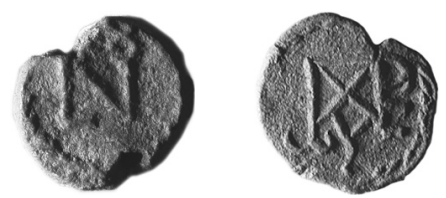

Datation : $\mathrm{VI}^{\mathrm{e}}$ siècle/VII ${ }^{\mathrm{e}}$ siècle.

\section{Théodore}

Inv. : 2001.28.

Dia. : 23,1. Sceau échancré aux deux extrémités du canal (particulièrement à l'extrémité inférieure), légèrement rogné et bien frappé.

Inédit. 
$\mathrm{Au}$ droit, monogramme invocatif (peut-être type Zacos et Veglery XXVIII) ; cercle de feuilles.

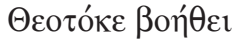

Au revers, monogramme cruciforme avec $\Theta$ à gauche, $\epsilon$ à droite, $P$ et $V$ au sommet, $\omega$ et $\Delta$ à la base; cercle de feuilles.

$\Theta \in O \triangle \omega P \gamma$

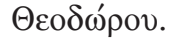
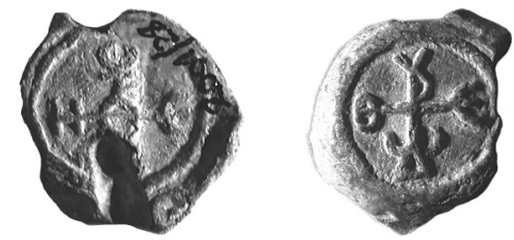

Datation : $\mathrm{VI}^{\mathrm{e}} / \mathrm{VII}^{\mathrm{e}}$ siècle.

\section{Constantin...}

Inv. : 4.1.000.

Dia. : 20,74. Sceau légèrement échancré aux deux extrémités. Gravure grossière. Parallèle : Zacos (BnF) 2970. ${ }^{10}$

Inédit.

Au droit, monogramme invocatif (type Zacos et Veglery XXV) ; dans les cantons, une étoile ; cercle de feuilles.

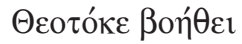

Au revers, inscription sur trois ou quatre lignes ; peut-être une étoile ; cercle de feuilles.

KWN|CTAN|TINOV|CTP

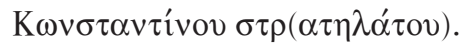
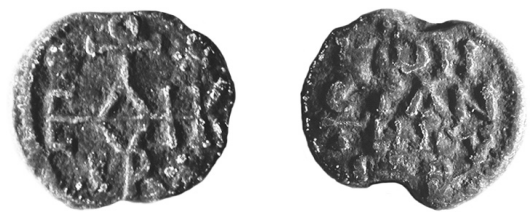

Datation : $\mathrm{VII}^{\mathrm{e}}$ siècle.

10 Jean-Claude Cheynet m'a signalé ce sceau dont le revers se transcrit ainsi : .WN|.TAN|TINOV|CTP, . 


\section{Georges}

Inv. : 1.53 .98 .

Dia. : 22,7. Sceau échancré et rogné.

Inédit.

Au droit, monogramme invocatif (type Zacos et Veglery XLVI) ; cercle de feuilles.

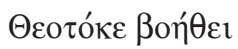

Au revers, monogramme cruciforme avec $E$ à droite, $P$ au centre, $\succ$ au sommet, probablement $\omega$ à la base ; cercle de feuilles ; frappe décentrée.

ГЄWPГI૪

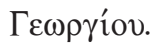
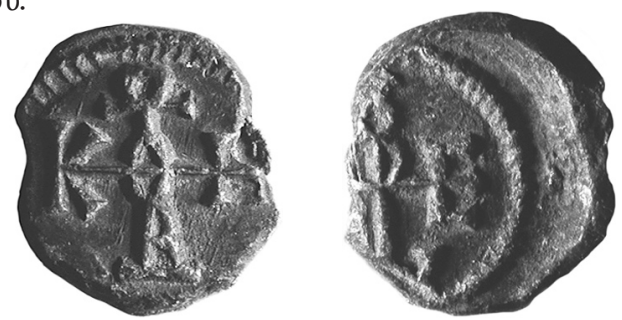

Datation : VII ${ }^{\mathrm{e}}$ siècle.

\section{Georges, apo hypatôn}

Inv. : 2.6.000.

Dia. : 21,06/22,26. Sceau échancré au droit aux extrémités du canal et rogné dans la partie droite. Rotation verticale.

Inédit.

$\mathrm{Au}$ droit, monogramme cruciforme avec $\Gamma$ à gauche, $\mathrm{E}$ à droite, $\mathrm{P}$ au centre, $\varnothing$ au sommet (la partie supérieure étant illisible), $\omega$ à la base ; cercle de feuilles.

ГЕ

$\Gamma \varepsilon \omega \rho \gamma$ íov

Au revers, inscription sur trois lignes ; cercle de feuilles.

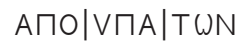

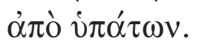
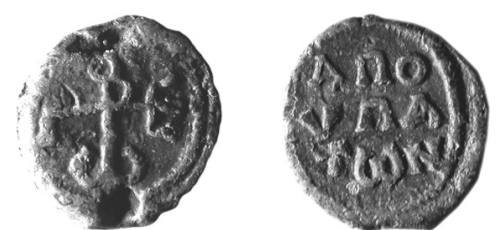

Datation : VII ${ }^{\mathrm{e}}$ siècle. Voir SBS 8 (2003), 237 (Münz, vente 94, 13-15 mai $\left.1998, n^{\circ} 917\right)$ : la pièce, datée de $610 / 650$, est décrite en des termes similaires. 


\section{Paul, commerciaire}

Inv. : 1.52.98.

Dia. : 20,9. Sceau rogné et un peu écrasé. Frappe décentrée vers la partie supérieure. Parallèle : Musée de Chypre 219.

Éd. : Metcalf, Cyprus, no 219 (pièce dont l'authenticité est considérée comme douteuse).

Au droit, inscription sur deux lignes, précédée d'une croisette :

$+\sqcap A \mid$ | $\vee \wedge O \bigvee$

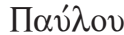

Au revers, inscription sur deux ou trois lignes, cercle de feuilles ; frappe décentrée :

-...PKIA|PIOV

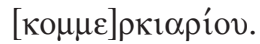
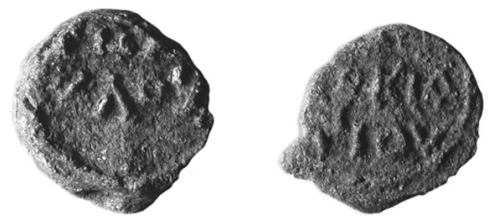

Datation : VII ${ }^{\mathrm{e}}$ siècle.

\section{Phôtinos, stratélate}

Inv. : 4.2.000.

Dia. : 20,7. Sceau légèrement échancré aux deux extrémités.

Inédit.

Au droit, monogramme cruciforme avec $\Phi$ à gauche, $N$ à droite, $T$ et $\zeta$ au sommet, $\omega$ à la base ; cercle de feuilles :

QUTIN乙

$\Phi \omega \tau$ เินขั

Au revers, inscription sur trois lignes ; croix dans le champ en haut ; cercle de feuilles :

$+\mid$ СTPA $\mid$ TH $\wedge$ A|TOV

$+\sigma \tau \rho \alpha \tau \eta \lambda \alpha ́ \tau o v$.
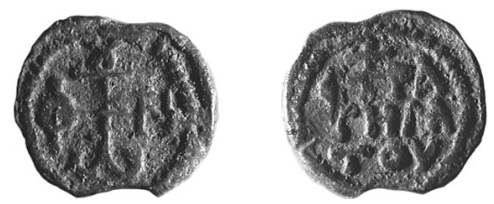

Datation : VII ${ }^{\mathrm{e}}$ siècle. 
10. N...

Inv. : 1.47.98.

Dia. : 26,8. Sceau cassé aux deux extrémités du canal et rogné.

Inédit.

$\mathrm{Au}$ droit, monogramme invocatif (proche du type Zacos et Veglery XXXVI, auquel il faut ajouter un $\mathrm{H}$ sur la barre horizontale droite avant le $\in$ ) ; cercle de feuilles.

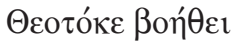

Au revers, monogramme cruciforme avec, de manière assurée, $\Pi$ et $\mathrm{N}$ à gauche, $X$ à droite, $\omega$ et $\wedge$ ou $A$ à la base, $\measuredangle$ au sommet, et, peut-être, $\Gamma$ au centre ; cercle de feuilles.
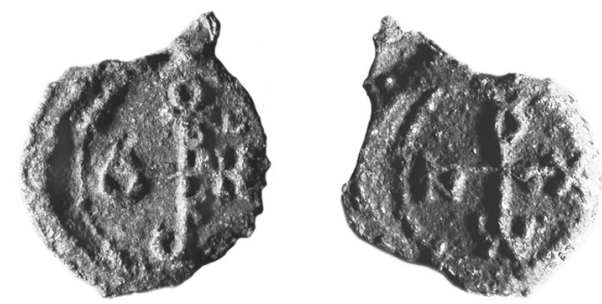

Datation : VII ${ }^{\mathrm{e}}$ siècle.

\section{N., apo éparchôn}

Inv. : 1.51.98.

Dia. : 19,89. Sceau échancré au droit aux deux extrémités du canal et rogné, particulièrement sur la gauche (au droit).

Inédit.

Au droit, monogramme invocatif avec $\Theta$ à gauche, I et $E$ à droite, $K$ en haut en position intermédiaire, auquel il faut ajouter $T$ et $O, B$ à la base :

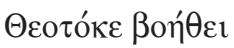

Au revers, inscription sur trois ou quatre lignes :

.AПỌ|.ПАРX|بN

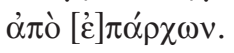
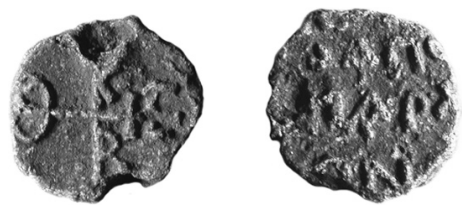

Datation : VII ${ }^{\mathrm{e}}$ siècle. 


\section{Georges, stratélate}

Inv. : 1.49.98.

Dia. : 27,66. Sceau échancré aux deux extrémités du canal. Revers corrodé. Inédit.

Au droit, monogramme invocatif (type Zacos et Veglery XXXV) ; cercle de feuilles :

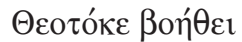

Au revers, inscription sur quatre lignes ; cercle de feuilles :

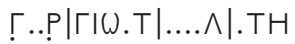

$\Gamma[\varepsilon \omega] \rho \gamma i \underline{\omega}[\sigma] \tau[\rho \alpha \tau \eta] \lambda[\alpha ́] \tau \eta ̣$
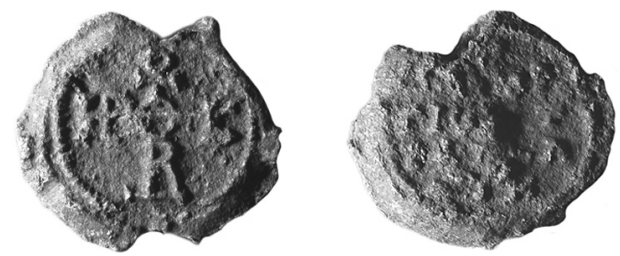

Datation : fin $\mathrm{VII}^{\mathrm{e}}$ siècle/début VIII ${ }^{\mathrm{e}}$ siècle.

\section{Jean, évêque de Faustinoupolis}

Inv. : 91.98 .

Dia. : 25,44. Sceau bien centré, mais légèrement pressé sur les deux faces. Inédit.

Au droit, inscription sur quatre lignes, précédée d'une croisette ; cercle de feuilles :

$$
\begin{aligned}
& \text { + IN|AMN V }|\in \Pi I C| \text { К ОП }
\end{aligned}
$$

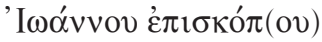

Au revers, inscription sur quatre lignes ; cercle de feuilles :

中AU|CT!̣૪|חO $\wedge \in \mid \omega C$

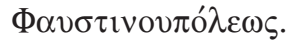
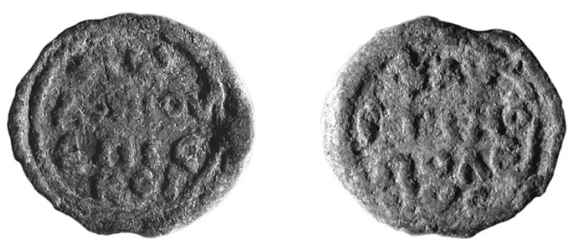

Datation : 650-750. L'évêché de Faustinoupolis est un évêché de la province de Cappadoce Seconde, suffragant de la métropole de Tyane. Bien qu'il soit attesté dans les Notices épiscopales $(1-4,7,9,10,13$, soit 
toutes les listes développées), ${ }^{11}$ seuls trois de ses titulaires sont connus, Daniel au concile d'Éphèse (431), Patrophilos au concile de Chalcédoine (451), Jean au concile de Constantinople III (680-681) et au concile in Trullo (691-692). Le propriétaire homonyme du sceau est identifiable à ce dernier. L'agglomération de Faustinoupolis est située à mi-chemin entre Podandos et Tyane, sur l'une des routes qui conduisent des Pyles ciliciennes au sud de la Cappadoce, depuis Tarse ou Adana jusqu'à Césarée, Ancyre ou Ikonion. ${ }^{12}$ Exposée aux expéditions arabo-musulmanes, elle aurait été à une date précoce abandonnée au profit des sites défensifs voisins, notamment celui de Loulon. En l'absence de témoignages textuels Friedrich Hild et Marcell Restle ne précisent pas la date de la désaffection du site. ${ }^{13}$ Les expéditions arabes contre la Cappadoce sont mentionnées dès le milieu du VII ${ }^{\mathrm{e}}$ siècle. ${ }^{14}$ Cités et places fortes de Cappadoce méridionale, au sortir des Pyles ciliciennes, sont signalées nommément à partir du début du VIII ${ }^{\mathrm{e}}$ siècle. ${ }^{15}$ La région au nord des Pyles ciliciennes commença en effet à être systématiquement attaquée une fois que les Arabes réussirent à s'établir durablement en Cilicie. Ce fut après l'installation de ces derniers à Mopsueste, où ils construisirent une mosquée et un grenier en 703, que Tyane fut prise par les Arabes en 707-708, puis abandonnée par ses habitants. ${ }^{16}$ La région de Tyane fut de nouveau attaquée avec succès par les Arabes en $739 / 740 .{ }^{17}$ Alors que les évêques du ressort de la métropole de Tyane, dans la Cappadoce la plus méridionale, participèrent aux conciles de la fin du VII ${ }^{\mathrm{e}}$ siècle, ${ }^{18}$ à l'exception de celui de Kybistra, un siècle plus

11 Darrouzès, Notitiae, «Notitia 1, » $n^{\circ} 225$, p. 209, « Notitia 2, » $n^{\circ} 282$, p. 223, «Notitia 3, $\mathrm{n}^{\circ} 311$, p. 237, « Notitia 4, $\mathrm{n}^{\circ}$ 238, p. 255, « Notitia 7, » $\mathrm{n}^{\circ} 290$, p. 278 , « Notitia $9, » n^{\circ} 176$, p. 299 , «Notitia $10, » n^{\circ} 209$, p. 316 , «Notitia 13 , » $\mathrm{n}^{\mathrm{o}} 215$, p. 357.

12 Fr. Hild, Das byzantinische Strassensystem in Kappadokien (Vienne, 1977), 55.

13 Fr. Hild et M. Restle, Kappadokien (cité n. 1), 258-259.

14 R.-J. Lilie, Die Byzantinische Reaktion auf die Ausbreitung der Araber (Munich, 1976), 61, 68. Sur l'implantation des Arabes en Cilicie et leurs expéditions au nord du Taurus, voir S. Métivier, «L'organisation de la frontière arabo-byzantine en Cappadoce ( $\mathrm{VIII}^{\mathrm{e}}-\mathrm{IX}^{\mathrm{e}}$ siècle), » Puer Apuliae. Mélanges offerts à Jean-Marie Martin, éd. E. Cuozzo, V. Déroche, A. Peters-Custot et V. Prigent (Paris, 2008) (Centre de recherche d'histoire et civilisation de Byzance, Monographies, 30), II, 433-454.

15 Lilie, Die Byzantinische Reaktion (cité n. 12), 114-122.

16 Théophane, Chronographia, AM 6201, éd. C. de Boor (Leipzig, 1883), I, 376-377.

17 Ibidem, AM 6231, I, 411.

18 Il s'agit, au concile de 680-681, des évêques de Tyane et de Faustinoupolis (absences des évêques de Kybistra et de Sasima), en 692, des évêques de Tyane, de Faustinoupolis et de Sasima ; à cette dernière date n'est absent que l'évêque de Kybistra. Voir E. Ohme, Das Concilium Quinisextum und seine Bischofsliste. Studien zum Konstantinopeler Konzil von 692 (Berlin, 1990), 259. 
tard n'assista au concile de Nicée II que celui de Sasima, au nord de Tyane. Le métropolite de Tyane y fut représenté, les évêques de Faustinoupolis et de Kybistra en furent absents. ${ }^{19}$ La conservation du sceau de Jean évêque de Faustinoupolis dans la région même de Faustinoupolis atteste la résidence de l'évêque Jean dans son évêché. Puisqu'on ne connaît pas d'exemples de sceaux frappés par des évêques sous juridiction arabe, on peut en déduire qu'à une date mal assurée l'évêché était effectivement pourvu et occupé, dans le ressort de l'Empire. Alors qu'au concile In Trullo les évêques déplorèrent l'abandon de leur église par les clercs et l'impossibilité pour certains de leurs collègues de gagner leur siège, en raison des expéditions des barbares (canons 18 et 37), ${ }^{20}$ on constate que la hiérarchie épiscopale était encore présente en Cappadoce méridionale à la fin du $\mathrm{VII}^{\mathrm{e}}$ siècle, voire au début du VIII ${ }^{\mathrm{e}}$ siècle.

\section{Georges, patrice}

Inv. : 8.1 .83 .

Dia. : 23,9. Sceau légèrement échancré aux extrémités du canal et rogné dans la partie supérieure.

Inédit.

Au droit, monogramme invocatif (type Zacos et Veglery XLIV) inscrit dans les quatre cantons au datif.

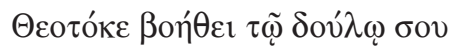

$\mathrm{Au}$ revers, monogramme cruciforme inscrit dans les quatre cantons, avec $€$ à gauche, $P$ à droite, $\omega$ à la base. Dans les quatre cantons,

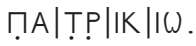

$\Gamma \varepsilon \omega \rho \gamma i \underline{\omega} \pi \alpha \tau \rho \imath \kappa i \omega$.
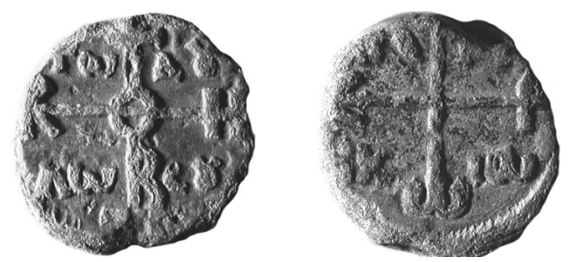

Datation : première moitié du $\mathrm{VIII}^{\mathrm{e}}$ siècle.

19 E. Lamberz, Die Bischofslisten des VII.Ökumenischen Konzils (Nicaenum II), (Munich, 2004), 43, 65. Le métropolite de Tyane est représenté par Nicolas higoumène du monastère d'Apros. Il est mentionné sur toutes les listes sauf sur F, considérée par E. Lamberz comme la plus fiable.

20 Discipline générale antique (II $\left.I^{e}-I X^{e} s.\right)$, t. I, 1 : Les canons des conciles cecuméniques, éd. et tr. fr. P.-P. Joannou, Grottaferrata (Rome, 1962) (Pontificia commissione per la redazione del codice di diritto canonico orientale. Fonti IX), 149-150, 171-172. 


\section{Jean Comnène Maurozômès}

Inv. : 1.54 .98 .

Dia. : 34,8 . Sceau cassé à gauche du champ et percé.

Inédit.

$\mathrm{Au}$ droit, saint de face, en pied, barbu, un cartel dans la main droite.

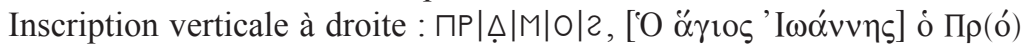

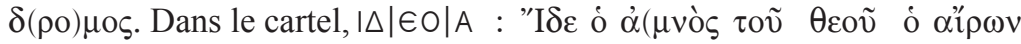

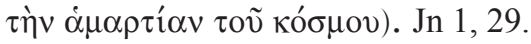

Au revers, inscription sur cinq lignes. Ligature à la ligne $4 \mathrm{du} Y$ et du P ; ligature à la ligne $5 \mathrm{du} T$ et du $\mathrm{P}$ :

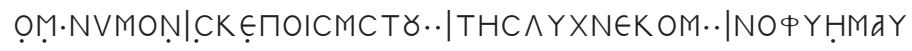

POTO|MHNПגTPOOEN

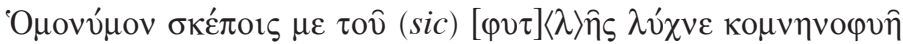

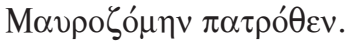

Je propose une autre lecture de la deuxième ligne que celle qui a été

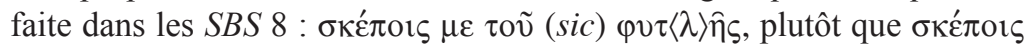
$\mu \alpha ́ \rho \tau \nu \varsigma \varphi v \tau\langle\lambda\rangle \hat{\eta}$.
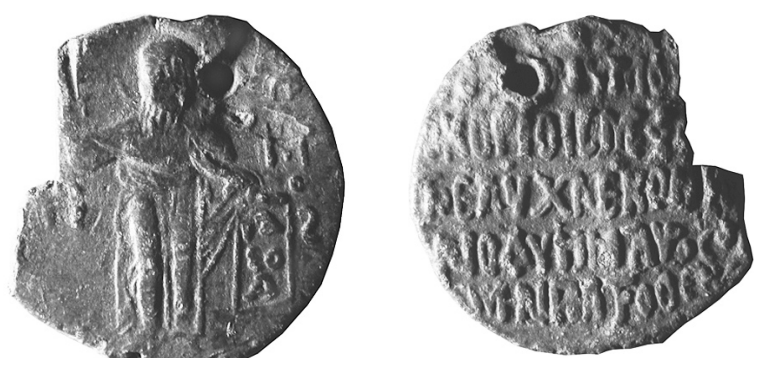

Datation : XIII ${ }^{\mathrm{e}}$ siècle. Le détenteur du sceau est identifiable avec le Jean Comnène Maurozômès mentionné dans une épitaphe grecque de 1297 conservée au musée archéologique de Konya. Il peut s’agir du fils de Manuel Maurozômès, connu, au début du $13^{\mathrm{e}}$ siècle, comme dignitaire du règne d'Alexis III et beau-père du sultan Kaykhusraw $\mathrm{I}^{\text {er }}$. Pour un commentaire plus détaillé sur Jean Comnène Maurozômès et sa famille, cf. mon article « Les Maurozômai, Byzance et le sultanat de Rūm. Note sur le sceau de Jean Comnène Maurozômès », REB 67 (2009), 197-207.

Le principal intérêt de cet ensemble sigillographique réside dans la conservation des sceaux dans les musées de Cappadoce. Une fois contextualisés, leur signification historique est précisée. Ils confirment par ailleurs le caractère local des sceaux, c'est du moins ce que prouvent le sceau du métropolite Eugénios de Césarée ainsi que celui de l'évêque Jean de Faustinoupolis. On remarque enfin l'absence d'anthroponymie proprement cappadocienne. 